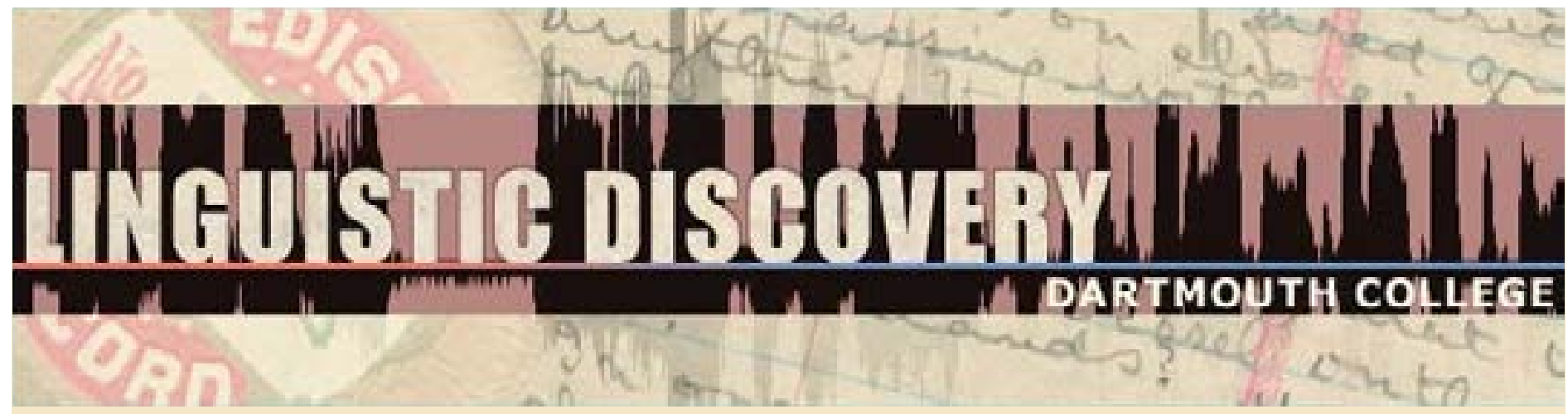

Volume 8 Issue 1 2010
Commentary on Boye - Semantic Maps as Helpers in the Quest for Generic Categories

Ljuba Veselinova

Stockholm University

doi: $10.1349 / P S 1.1537-0852 . A .360$

url: http://journals.dartmouth.edu/cgi-bin/WebObjects/ Journals.woa/1/xmlpage/1/article/360
Linguistic Discovery

Published by the Dartmouth College Library Copyright to this article is held by the authors. ISSN 1537-0852 linguistic-discovery.dartmouth.edu 


\title{
Semantic Maps as Helpers in the Quest for Generic Categories
}

\author{
Comment on 'Semantic Maps and the Identification of Cross-Linguistic \\ Generic Categories: Evidentiality and its Relation to Epistemic Modality' by \\ Kasper Boye (2010)
}

\author{
Ljuba Veselinova \\ Stockholm University
}

Boye (2010) addresses a very important point, namely, the definition of the concept "category" in functionally-oriented cross-linguistic theory. The notion of a category becomes a thorny issue any time some serious theoretical reasoning is attempted in cross-linguistic work. What becomes immediately apparent during such theorizing is not only the complete lack of generally accepted definitions of the concept "category" but also, very often, widespread unawareness of this deficiency. Boye's attending to this inadequacy, in turn, makes his paper a true contribution to linguistic theory. The author argues that semantic maps are a necessary, though not necessarily sufficient, way of defining the notion "generic category" in a coherent manner. To illustrate his point, Boye presents a semantic analysis of evidentiality and epistemic modality. The theoretical reasoning is empirically grounded in a fairly well-spread typological sample of 55 languages, through which the author aims to elucidate the core senses of the grams studied.

However, I have a question regarding the methodological justification for utilizing semantic maps in general, or their raison d-être, as it were. What are the reasons for assuming that semantic maps provide a better approach to defining the notion "category" than, say, prototype theory? Furthermore, Boye tends to label generalizations as "trivial" or "non-trivial" very frequently throughout the paper. In fact, one is left with the impression that a substantial part of his argumentation is actually based on classifying various facts according to their "triviality". It is not always clear, however, where he draws the line between trivial and non-trivial matters. I think that an explicit definition of this distinction would be needed.

\section{Reference}

Boye, Kasper. 2010. Semantic maps and the identification of cross-linguistic generic categories: Evidentiality and its relation to Epistemic Modality. Linguistic Discovery, this issue.

Author's contact information:

Ljuba Veselinova

Department of Linguistics

Stockholm University

S-106 91 Stockholm

Sweden

ljuba@ling.su.se 